\title{
Fermilab
}

\section{Accelerating Heavy Ions in Fermilab}

L. C. Teng

November 1983

Recently several laboratories ${ }^{1}$ initiated discussions on the design and construction of high energy heavy-ion colliders to study the formation and the characteristics of quark/gluon plasma. The beam energies considered are $>30 \mathrm{GeV} /$ nucleon and the costs of the machines considered are all in the hundreds of megabuck class. We consider here a poor-man's scheme of entering into a first phase of such studies.

The Fermilab Tevatron can be modified to accelerate heavy ions which can be used to strike fixed targets. Assuming that the Tevatron can accelerate protons to $1 \mathrm{TeV}$, it can, then, accelerate $\mathrm{U}_{238}^{92+}$ (say) to $386 \mathrm{GeV} /$ nucleon. When striking a stationary $U$ ion this is equivalent to colliding beams of beam energy $12.6 \mathrm{GeV} /$ nucleon. Although this is less than $30 \mathrm{GeV} /$ nucleon it is high enough to be of interest and should give a strong indication on whether a step toward higher energy should be taken.

For the present discussion it is adequate and, indeed, more illuminating to approximate the mass of an ion with mass number $A$ by $A x$ (proton mass). We will also understand by the momentum and the energy of an ion the momentum per nucleon and the energy per nucleon. We shall never need to talk about the momentum and the energy of the whole ion.

When a proton synchrotron is used to accelerate heavy ions the operating ranges of the $\mathrm{rf}$ frequency and the guide field strength will correspond to different momentum ranges of the ion. The $r f$ will operate for ions over the same momentum range (same $\beta$ range) as for protons but the guide field will 
operate over a momentum range of the ion equal to $\frac{Z}{A}$ times that of the proton (same rigidity $B \rho$ range) ( $Z$ = charge number, $A=$ mass number). Without modifying at least one of these systems the momentum range over which the ion can be accelerated is rather severely limited. The principal objective of this investigation is, thus, to find the most expedient and economical scheme, namely to decide what systems to modify and what systems to add. The operable momentum ranges of the various Fermilab synchrotrons for proton and for uranium ion are

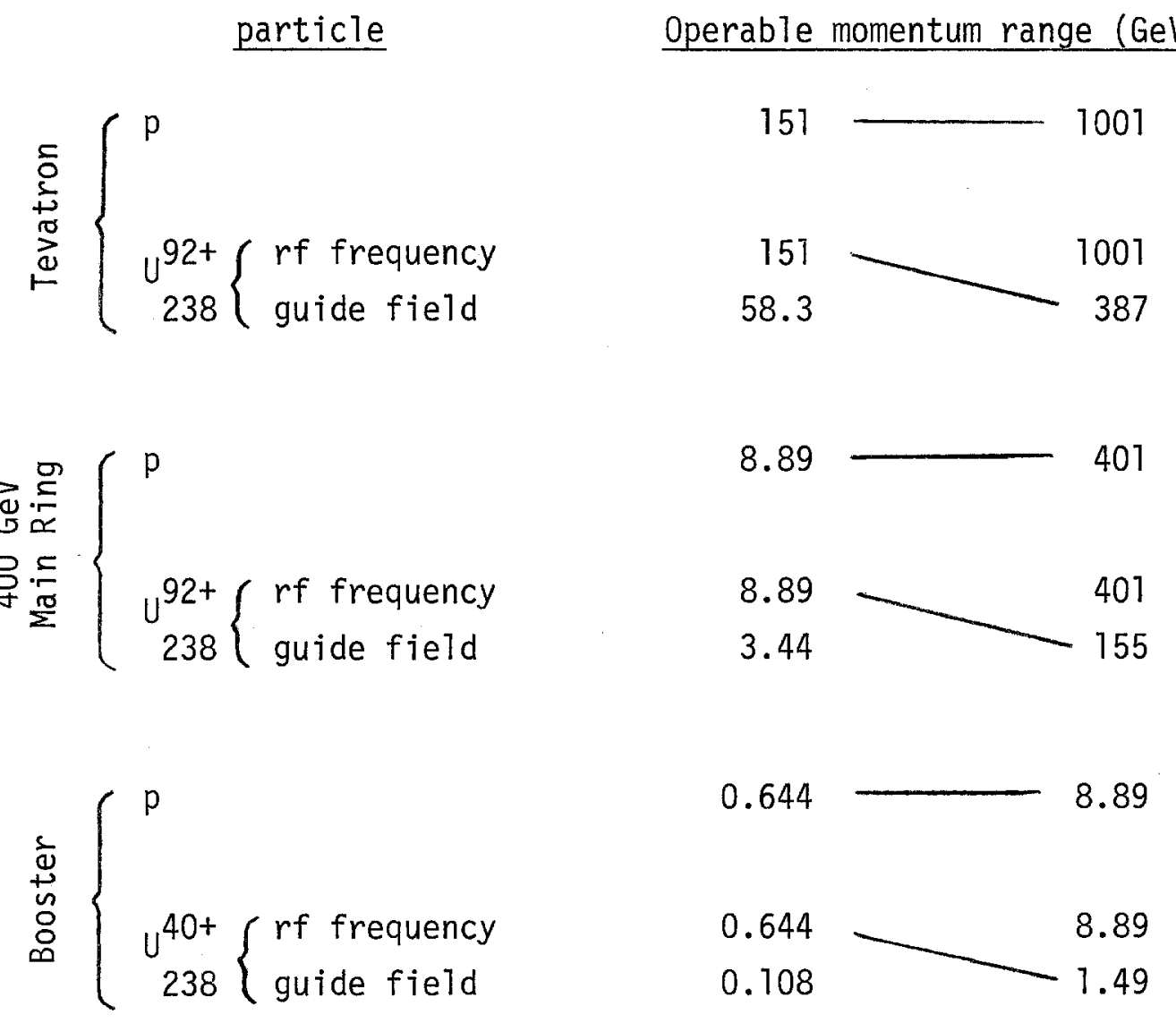

In this table $\mathrm{U}^{40+}$ is assumed for the Booster because below $\sim 1.5 \mathrm{GeV} / \mathrm{c}$ the efficiency of stripping to $\mathrm{U}^{92+}$ is rather poor. From this table we conclude the following. 
1. Assuming that the " $400 \mathrm{GeV}$ proton capability of the Main Ring is retained (questionable with overpass), the top of the Main Ring range $(155 \mathrm{GeV} / \mathrm{C})$ does overlap the bottom of the Tevatron range $(151 \mathrm{GeV} / \mathrm{c})$.

2. If no modification is made to the Main-Ring $r f$ system one needs to add a synchrotron to bridge the gap between the Booster and the Main Ring. This synchrotron can be designated as

$$
\mathrm{U}_{238}^{92+} \quad 1.49 \mathrm{GeV} / \mathrm{c}-8.89 \mathrm{GeV} / \mathrm{C}
$$

and is equivalent to a $23 \mathrm{GeV} / \mathrm{c}$ proton synchrotron. Such a synchrotron wi11 cost more than $M \$ 60$.

3. It is more economical to modify the Main Ring rf system to extend the lower limit of the frequency range down to $\sim 45 \mathrm{MHz}$ corresponding to a momentum of $1.49 \mathrm{GeV} / \mathrm{c}$. Such an $\mathrm{rf}$ system may cost about $\mathrm{M} \$ 10$. The guide field of the Main Ring needs no modification to operate down to $172 \mathrm{G}$ for $\mathrm{U}^{92+}$ at $1.49 \mathrm{GeV} / \mathrm{c}$. With this modification one can inject the $1.49 \mathrm{GeV} / \mathrm{C}$ beam from the Booster (after stripping to $92+$ ) directly into the Main Ring.

4. It is difficult to conceive of extending the lower limit of the Booster $r f$ down farther. Thus we need a $0.644 \mathrm{GeV} / \mathrm{c} \mathrm{U}^{40+}$ injector into the Booster. A linac would be prohibitively expensive. It is equivalent to a $3 \mathrm{GeV}$ proton linac which may cost around $\mathrm{M} \$ 200$. Thus, we are forced to consider a 1 inac/synchrotron combination. In this case, it would make more sense and the logistics would be much simpler to accelerate the ions in this synchrotron all the way to $1.49 \mathrm{GeV} / \mathrm{c}$ (or slightly higher) and inject directly into the Main Ring instead of accelerating to $0.644 \mathrm{GeV} / \mathrm{c}$, extracting and injecting into the Booster and there, accelerating further to $1.49 \mathrm{GeV} / \mathrm{c}$. The $1.49 \mathrm{GeV} / \mathrm{c}$ synchrotron will cost more and require a larger rf frequency modulation than the $0.644 \mathrm{GeV} / \mathrm{c}$ synchrotron but the simplicity 
and the reduced interference derived from bypassing the Booster is well worth the cost. In any case, it is doubtful that the Booster vacuum can be sufficiently improved for accelerating non-fully-stripped ions. This has the added advantage that one can now choose a higher final momentum, say, $1.7 \mathrm{GeV} / \mathrm{c}$ at which the stripping efficiency to $92+$ is approaching 100\%. An appropriate energy for the linac is $10 \mathrm{MeV}$ or a momentum of $0.137 \mathrm{GeV} / \mathrm{c}$.

The entire scheme is now as follows:

$\underline{\text { Linac }}^{2}$ (new, M\$ 30) ( 100 m long)

\section{Section I}

\begin{tabular}{lc|c} 
Ion & $U^{5+}$ & $\frac{0}{5}$ \\
Energy & $0-1.2 \mathrm{MeV}$ & $\frac{0}{0}$ \\
\hline$\frac{2}{2}$ \\
Momentum & $0-47.5 \mathrm{MeV} / \mathrm{c}$ & w
\end{tabular}

Section II

$\mathrm{u}^{40+}$

$1.2 \mathrm{MeV}-10 \mathrm{MeV}$

4.75 MeV/c - $137 \mathrm{MeV} / \mathrm{c}$

Booster (new, $M \$ 40$ ) ( r same size as $8 \mathrm{GeV} \mathrm{p}$ Booster)

Ion $u^{40+}$

Momentum

$0.137 \mathrm{GeV} / \mathrm{c}-1.70 \mathrm{GeV} / \mathrm{c}$

Energy

rf frequency

$10 \mathrm{MeV}-1 \mathrm{GeV}$

$7.673 \mathrm{MHz}-46.493 \mathrm{MHz}$

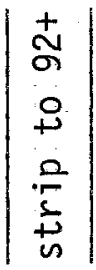

Main Ring (new rf system, M\$10)

Ion

$$
u^{92+}
$$

Momentum

$1.70 \mathrm{GeV} / \mathrm{c}-150 \mathrm{GeV} / \mathrm{c}$

Energy

$1 \mathrm{GeV}-149 \mathrm{GeV}$

rf frequency

$46.493 \mathrm{MHz}-53.104 \mathrm{MHz}$

Tevatron (no modification)

Ion

$$
u^{92+}
$$

Momemtum

$150 \mathrm{GeV} / \mathrm{C}-387 \mathrm{GeV} / \mathrm{C}$

Energy

$149 \mathrm{GeV}-386 \mathrm{GeV}$

rf frequency

$53.104 \mathrm{MHz}-53.105 \mathrm{MHz}$ 
The total cost is $\sim M \$ 80$. The construction and commissioning of the whole injector (Linac and Booster) and of the new Main Ring rf system can be carried out totally independent of the operation of the Tevatron complex. Downtime is required only to install the rf cavities in the Main Ring and to connect the beam pipe between the new Booster and the Main Ring. The injection and extraction systems in both the Main Ring and the Tevatron can be used without modification. If the work is properly organized, the interruption should not have to be more than $\sim 1$ month.

With a $230 \mu \mathrm{A}$ (particle) beam from the Linac one can inject $\sim 2 \times 10^{11}$ ions per Tevatron pulse. There will be losses during stripping, adiabatic capture, beam transfers between synchrotrons, etc.; but the overall efficiency should be better than $\frac{1}{2}$, i.e. one should be able to get more than $1 \times 10^{11}$ $\mathrm{u}^{92+} /$ pulse out of the Tevatron.

\section{References}

${ }^{1}$ Notably BNL and ORNL

${ }^{2}$ The design of a $230 \mu \mathrm{A}$ (particle), $10 \mathrm{MeV} /$ nucleon $\mathrm{U}^{40+} 1$ inac was made by LANL for ORNL. The important parameters are given in Table 1 .

${ }^{3}$ The study for one specific scheme was made by A.G. Ruggiero, "HeavyIon Beams for Fermilab", October 1983 
Table 1 LOS ALAMOS NATIONAL LABORATORY -- ACCELERATOR TECHNOLOGY DIVISION CONCEPTUAL HEAVY ION LINAC FOR OAK RIOGE

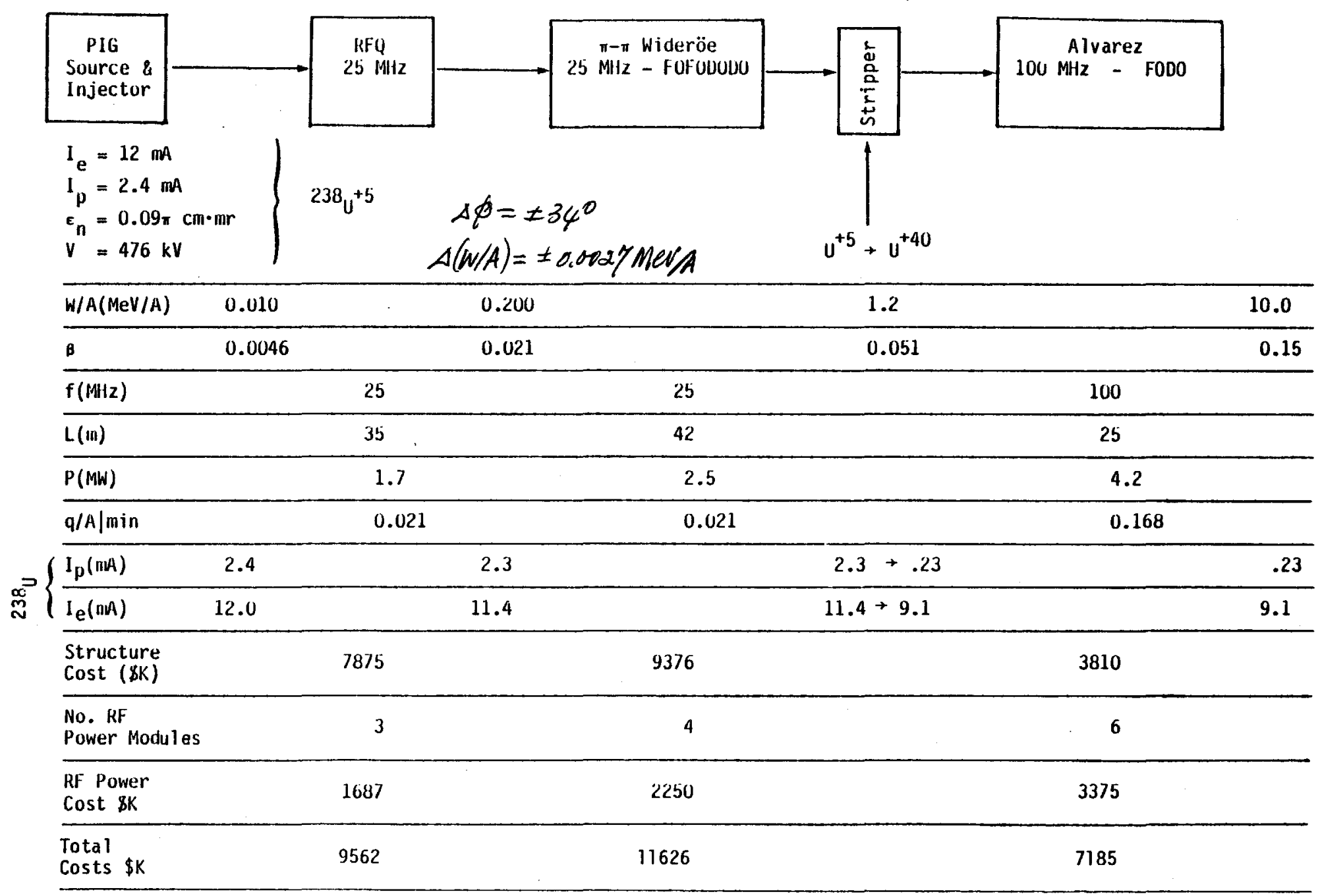

Debunched $\triangle(W / A)=\neq 0.00051 \mathrm{Mer} / \mathrm{A}$ (comerved durcin acceleration) for limac 